\title{
Overcoming a fast transverse instability by means of octupole-induced tune spread in the Relativistic Heavy Ion Collider
}

\author{
C. Montag, J. Kewisch, and D. Trbojevic \\ Brookhaven National Laboratory, Upton, New York 11973 \\ F. Schmidt \\ CERN, 1211 Geneva 23, Switzerland \\ (Received 11 July 2002; published 20 August 2002)
}

\begin{abstract}
During the Relativistic Heavy Ion Collider commissioning in 2001 a fast transverse instability was observed on the ramp. In general this could be counteracted with increased chromaticity, resulting in Landau damping. However this method could not be applied around transition energy where chromaticities have to change sign. So octupoles were used near transition energy to create transverse Landau damping and avoid the transverse instability, emittance blowup, and beam loss. This paper describes the considerations that led to the present scheme, as well as experimental results.
\end{abstract}

DOI: 10.1103/PhysRevSTAB.5.084401

PACS numbers: 29.27.Bd, 29.20.Lq

\section{INTRODUCTION}

The Relativistic Heavy Ion Collider (RHIC) is designed to accelerate and collide fully stripped gold ions at energies of up to $100 \mathrm{GeV}$ per nucleon, equivalent to a relativistic $\gamma$ factor of $\gamma=107$ [1]. Gold ions are injected from the Alternating Gradient Synchrotron (AGS) into the two RHIC rings ("Blue" and "Yellow") at $\gamma=10.5$ and then accelerated to storage energies. During the acceleration the transition energy of RHIC at $\gamma_{t}=23.23$ is crossed. This transition energy crossing can introduce many transverse and longitudinal problems such as space charge mismatch, transverse microwave instability, Robinson instability, and others.

The linear transition jump in RHIC was designed and built to minimize these transition crossing problems [2]. Since $\gamma_{t}$ is a lattice parameter, it can be slightly modified by transverse optics changes. This is accomplished by ramping a set of special quadrupoles before the beam energy approaches transition, which results in an increased value of $\gamma_{t}$. The current in the quadrupoles is then reversed within $35 \mathrm{msec}$ which jumps $\gamma_{t}$ to a value below the beam energy. The rf synchronous phase $\phi_{s}$ is changed (by $180^{\circ}-2 \phi_{s}$ ) at the same time. To fight the head-tail effect the chromaticites must change sign. However, the sextupoles have no jump mechanism and their strengths change rather slowly.

During the RHIC run in 2001 a fast instability was observed on the ramp, limiting the maximum total beam intensity to roughly $25 \times 10^{9}$ gold ions in 55 bunches - about half the design value. This instability resulted in fast beam loss (Fig. 1) and transverse emittance blowup and could also be observed in the longitudinal phase space (Fig. 2). While the cause of this instability is still unknown, its rise time indicates it to be a transverse rather than a longitudinal instability. Figure 3 shows three longitudinal bunch profiles taken by the wall current monitor, the time between profiles corresponding to 125 revolutions in RHIC. While the first profile is roughly Gaussian, the second one exhibits a "shoulder" on its right slope. The third profile shows a clear double-peak structure.

To overcome the intensity limitation imposed by this instability, chromaticities were increased to provide sufficient Landau damping. However, this method failed right at transition where the sign change - and thus small chromaticities - are required. It was quickly found that the necessary Landau damping could be provided by bringing the two beams into collision around transition energy, which raised the maximum achievable beam intensity to some $30 \times 10^{9}$ ions per beam.

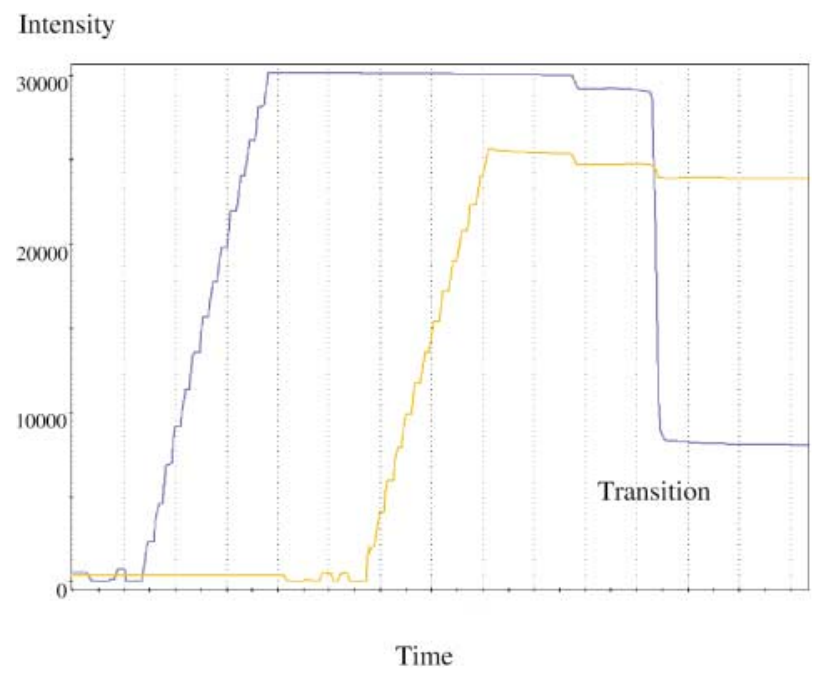

FIG. 1. (Color) Beam intensities in units of $10^{6}$ gold ions versus time during injection and ramp towards $100 \mathrm{GeV} /$ nucleon. While the blue beam is above the instability threshold and therefore suffers some $60 \%$ beam loss shortly after crossing $\gamma_{t}$, the yellow beam survives since it is below the respective threshold intensity. 


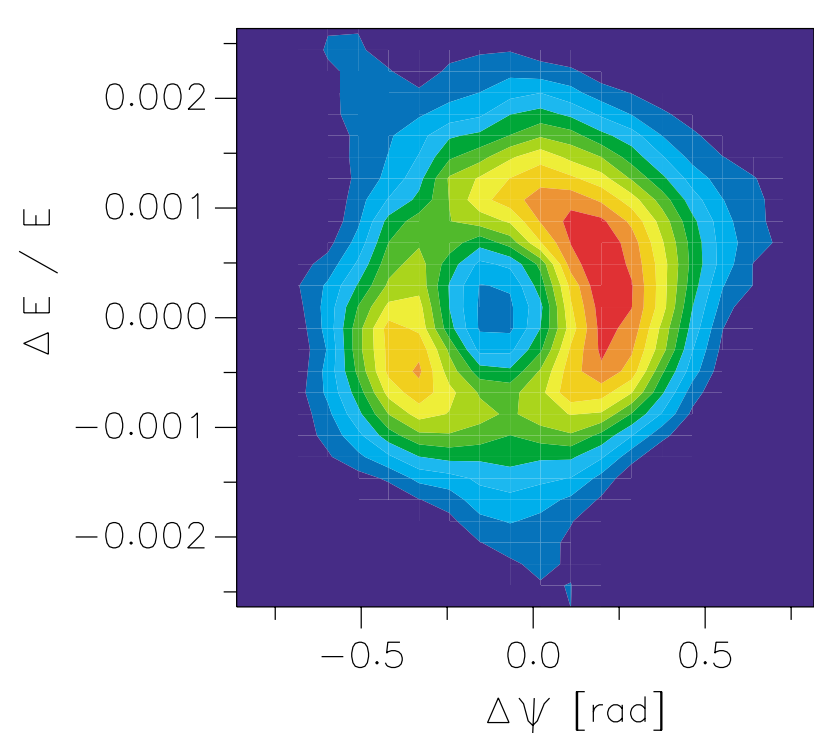

FIG. 2. (Color) Tomographic reconstruction of the longitudinal phase space distribution of a single bunch, $0.55 \mathrm{sec}$ after transition crossing. About $0.45 \mathrm{sec}$ after the transition jump the fast instability occurs, leading to a double-peak structure [3].

When attempts were made to further increase the beam current, large fractions of the beams were lost, presumably due to the beam-beam kick when both beams were brought into collision. Therefore an alternative method to provide a sufficient tune spread was developed, namely, the use of octupoles.

\section{DRIVING TERM CALCULATIONS}

The RHIC collider consists of two rings, each having six arcs with long straight sections. Out of 46 quadrupole magnets per arc, 11 are equipped with octupole coils. They are powered in four families, two families in the arcs with large dispersion and two families in the straight sections where the dispersion is small but nonzero due to the interaction region dipoles which provide head-on collisions.

Additionally, each of the 12 final focus quadrupole triplets is equipped with two additional octupole windings for local field compensation purposes. However, only triplet octupoles in two interaction regions (IRs) are connected to power supplies. Therefore only eight octupoles in the triplets were available during the experiments.

Because the tunes in RHIC are in the interval between 0.2 and 0.25 , the driving terms of the quarter resonance are of concern since they are driven to first order in the octupole strength. We therefore derived the driving terms of the $(4,0)$ resonance and the detuning coefficients of all octupole families. Resonance driving terms and detuning coefficients can be calculated in an automatic fashion using standard normal form tools [4] which need as an input a one-turn map derived with differential algebra [5]. These map creation and analysis tools are implemented in the tracking code SIXTRACK [6].

The procedure to find these resonances and detuning coefficients is as follows: one takes a MAD8 input file of RHIC, which includes the various octupole families; then a special MAD-8 version $[7,8]$ is run which produces the SIXTRACK input files; a subsequent SIXTRACK run produces
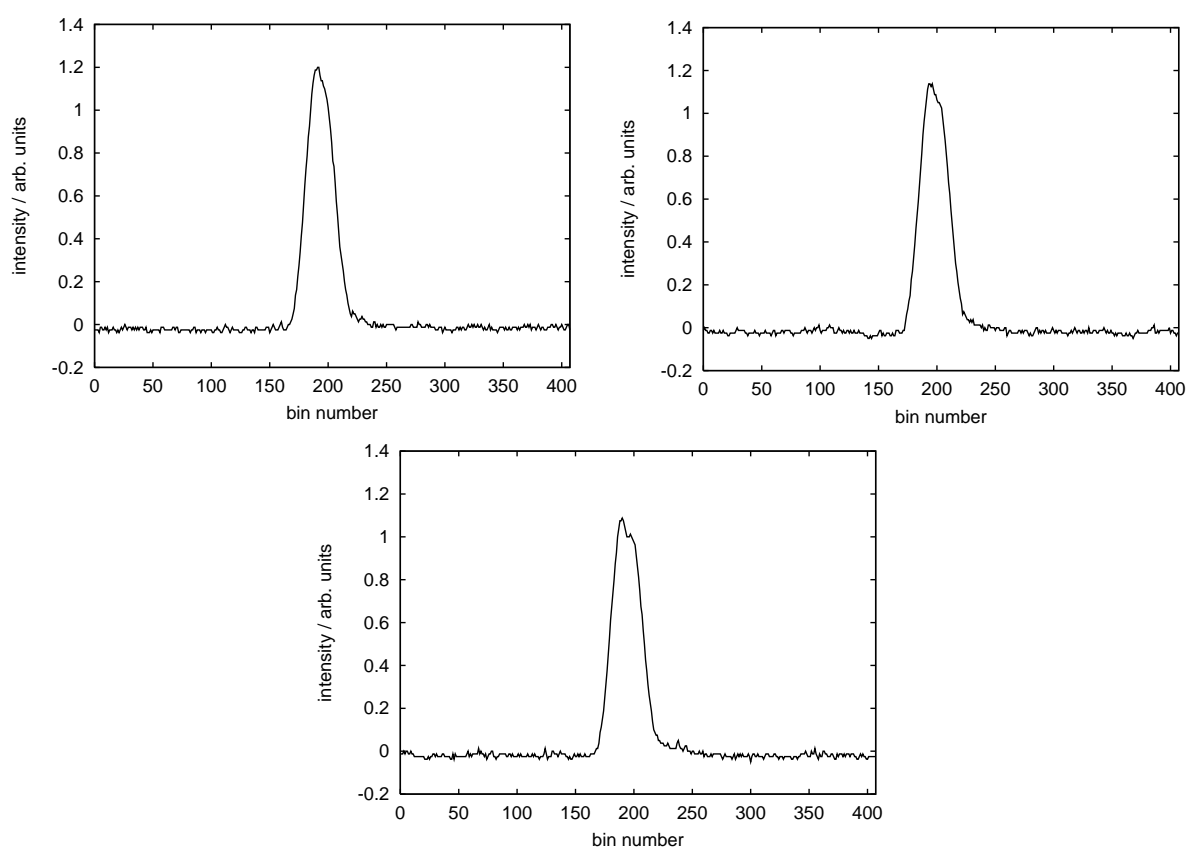

FIG. 3. Three consecutive longitudinal bunch profiles, taken every 125 turns $(1.6 \mathrm{msec})$. Since the initially Gaussian bunch profile (top) evolves into a double-peak structure (bottom) within $3.2 \mathrm{msec}$ ( 250 revolutions), this fast rise time may indicate a transverse rather than a longitudinal instability. 
TABLE I. Driving term $H_{40}$ of the $(4,0)$ resonance and horizontal detuning coefficient $\delta Q_{x} / \delta \epsilon_{x}$ per octupole family. The sum of the detuning coefficients of all eight triplet octupoles is about 2.7 times larger than the corresponding value for the four arc octupole families.

\begin{tabular}{|c|c|c|c|}
\hline \multirow{2}{*}{$\begin{array}{l}\text { Octupole } \\
\text { name }\end{array}$} & \multicolumn{2}{|c|}{$H_{40}$} & \multirow{2}{*}{$\begin{array}{c}\frac{\delta Q_{x}}{\delta \epsilon_{x}} \\
\left(10^{-3}\right)\end{array}$} \\
\hline & cos term $\left(10^{-3}\right)$ & sin term $\left(10^{-3}\right)$ & \\
\hline B3M05C1B & 0.17 & -0.15 & -0.85 \\
\hline B3M05C $3 B$ & 0.98 & -0.55 & -4.3 \\
\hline B3M06C1B & 0.70 & -0.05 & -2.7 \\
\hline B3М06C3B & 0.14 & -0.04 & -0.56 \\
\hline B3M07C1B & 0.57 & -0.42 & -2.7 \\
\hline B3M07C3B & 0.13 & -0.06 & -0.56 \\
\hline B3M08C1B & 0.16 & -0.15 & -0.85 \\
\hline B3М08C3B & 0.95 & -0.59 & -4.3 \\
\hline $\mathrm{OCT} 2 \mathrm{~F}$ & -0.017 & -0.05 & -4.2 \\
\hline OCT2D & 0.002 & -0.004 & -0.25 \\
\hline OCTF & -0.03 & -0.001 & -1.6 \\
\hline OCTD & -0.003 & -0.0007 & -0.21 \\
\hline
\end{tabular}

the one-turn map of RHIC; and last the map analysis is done with the DALIE code [9].

Table I shows that the lattice octupoles (last four entries in the table) have sufficiently strong detuning coefficients (last column) while the cosine and sine terms of the $(4,0)$ resonance (second and third columns) are much smaller than those of the octupoles located in the IRs (names starting with "B3M05C"). These four lattice octupole families are therefore the obvious choice for creating detuning without driving the fourth order resonances which would limit the dynamic aperture and cause particle losses.

\section{ESTIMATE AND MEASUREMENT OF REQUIRED OCTUPOLE STRENGTH}

Based on experience with colliding beams at transition energy, the required octupole strength was estimated as follows: In the case of round beams the beam-beam kick $\Delta z^{\prime}\left(=\Delta x^{\prime}\right.$ or $\left.\Delta y^{\prime}\right)$ can be expressed as [10]

$$
\begin{gathered}
\Delta z^{\prime}=\frac{Z^{2}}{A} \frac{2 N_{b} r_{p} z}{\gamma r^{2}}\left[1-\exp \left(-\frac{r^{2}}{2 \sigma^{2}}\right)\right], \\
r^{2}=x^{2}+y^{2} .
\end{gathered}
$$

Here $Z=79$ is the charge state of the gold ions, $A=$ 197 is the number of nucleons, and $N_{b}$ is the number of ions per oncoming bunch. $r_{p}=1.84 \times 10^{-18} \mathrm{~m}$ denotes the classical proton radius, while $\sigma=0.6 \mathrm{~mm}$ is the rms beam size at the interaction point for a design normalized emittance of $\epsilon_{n}=10 \pi \mathrm{mm} \mathrm{mrad}$, and $\beta_{x}^{*}=\beta_{y}^{*}=\beta^{*}=$ $5 \mathrm{~m}$ during transition crossing. The Lorentz factor is $\gamma=$ $\gamma_{t}=23.23$.

Expanding the exponential leads to

$$
\Delta z^{\prime}=\frac{Z^{2}}{A} \frac{2 N_{b} r_{p} z}{\gamma r^{2}}\left[1-\sum_{n} \frac{\left(\frac{x^{2}}{2 \sigma^{2}}\right)}{n !}\right]
$$

thus the octupole term in the horizontal plane $(z=x, y=$ $0)$ can be written as

$$
\Delta x_{\mathrm{oct}}^{\prime}=\frac{Z^{2}}{A} \frac{2 N_{b} r_{p}}{\gamma} \frac{1}{2 \times 4 \sigma^{4}} x^{3},
$$

which corresponds to an integrated octupole strength of

$$
o_{\mathrm{bb}}=\frac{Z^{2}}{A} \frac{2 N_{b} r_{p}}{\gamma} \frac{1}{2 \times 4 \sigma^{4}} .
$$

A similar expression exists for the vertical plane $(z=y$, $x=0$ ).

For an octupole located at a position where $\beta=\beta_{\text {oct }}$, the integrated strength $o=o_{\text {oct }}$ required to produce the same tune shift as the beam-beam interaction scales as

$$
o_{\text {oct }}=o_{\mathrm{bb}} \frac{\beta^{* 2}}{\beta_{\mathrm{oct}}^{2}} .
$$

Since beam-beam tune spread was sufficient to fight the fast instability for intensities up to some $N_{b}=$ $25 \times 10^{9} / 55$ gold ions per bunch, octupoles have to provide a similar tune shift. The integrated beam-beam octupole term per interaction point can be calculated according to Eq. (4) as $o_{\mathrm{bb}}=2.2 \times 10^{3} \mathrm{~m}^{-3}$; this has to be scaled with the $\beta$ function at the location of the respective octupoles [Eq. (5)].

If IR octupoles were to be used, the required strength can be easily estimated, using the considerations given above. Octupoles in the interaction region triplets are located at a distance of about $s=30 \mathrm{~m}$ from the interaction point, therefore the $\beta$ function at their location is about $\beta_{\text {oct }}=\beta^{*}+\frac{s^{2}}{\beta^{*}}=185 \mathrm{~m}$. If the beam-beam detuning of a single interaction point were to be provided by a single IR octupole, the required integrated octupole strength would therefore be $o_{\text {oct }}=1.6 \mathrm{~m}^{-3}$. In the case of RHIC, eight IR octupoles are available to provide the tune shift corresponding to six beam-beam interaction points; thus the minimum required integrated IR octupole strength is estimated at $o_{\text {oct }}=1.2 \mathrm{~m}^{-3}$. This strength has to be increased by a factor of 2.7 for the arc octupoles because of the much smaller $\beta$ functions at their locations; see Table I.

\section{EXPERIMENTAL RESULTS}

As Table I shows, for a given detuning the contribution of arc octupoles to the width of the quarter resonance is about 2 orders of magnitude smaller than for the case of IR octupoles. This scheme was therefore expected to be more beneficial in terms of maximum achievable beam intensities. Though the number of octupoles involved in this scheme is much larger than the number of IR octupoles, their sum effect on the beam tune spread is smaller by a factor of 2.7 due to the much smaller $\beta$ functions. The integrated octupole strength was therefore set to $o=3.0 \mathrm{~m}^{-2}$ for all four arc octupole families.

Using arc octupoles leads to a substantial increase in attainable beam intensities, as Fig. 4 shows. At intensities close to $40 \times 10^{9}$ gold ions per beam, only some $5 \%$ 


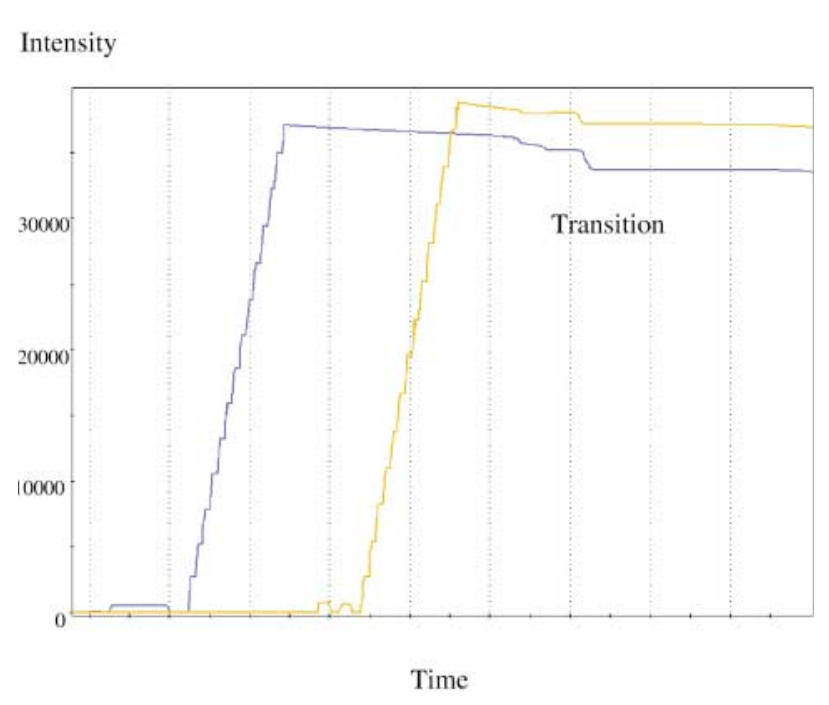

FIG. 4. (Color) Beam intensities in units of $10^{6}$ gold ions versus time during injection and on the ramp, with arc octupoles on. Though intensities are about $35 \times 10^{9}$ and $37 \times 10^{9}$ gold ions per beam, respectively, both beams suffer only about $5 \%$ loss around transition.

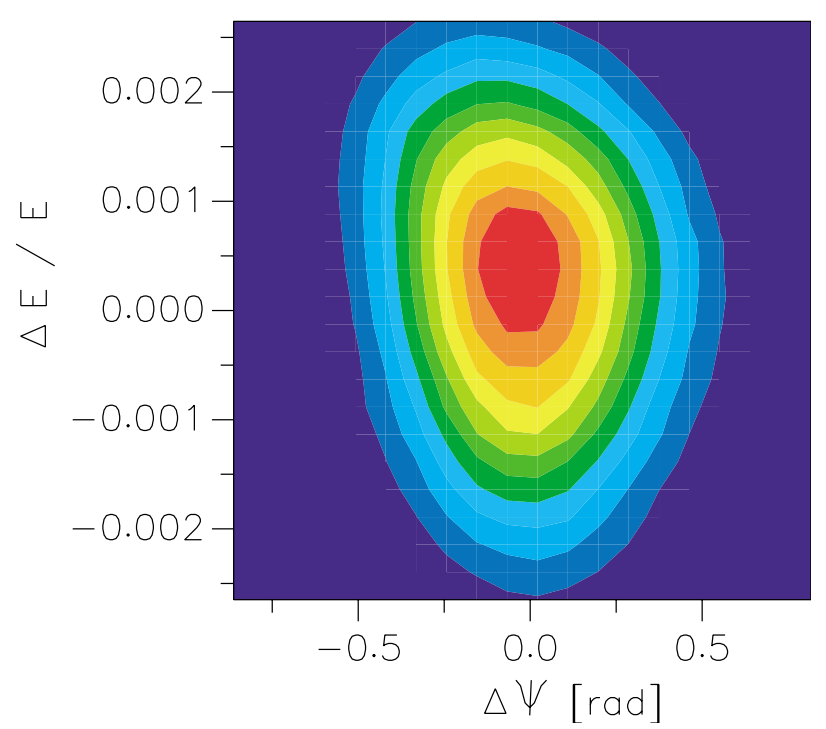

FIG. 5. (Color) Tomographic reconstruction of the longitudinal phase space distribution of a single bunch, $0.55 \mathrm{sec}$ after transition crossing, with octupoles on. No instability occurs. beam loss occurs at transition. Additionally, neither transverse emittance blow-up nor longitudinal bunch distortions (Fig. 5) were observed, clearly excluding the fast instability as the root cause of this remaining beam loss.

\section{CONCLUSION}

A fast instability around transition energy has been successfully counteracted by means of octupole-induced Landau damping; this damping could not be provided by increased chromaticity due to the required chromaticity sign change at transition. The optimum integrated octupole strength is in good agreement with estimates derived from beam-beam detuning, which was initially used for the same purpose.

\section{ACKNOWLEDGMENTS}

We would like to thank T. Roser for stimulating and encouraging discussions, and V. Ptitsyn for his help tuning the machine. We are also indebted to $\mathrm{H}$. Grote for his software support, as well as to T. Satogata for reading the manuscript. This work was performed under the auspices of the U.S. Department of Energy.

[1] RHIC design manual.

[2] S. Peggs, S. Tepikian, and D. Trbojevic, in Proceedings of the PAC 1993, Washington, D.C. (IEEE, Piscataway, NJ, 1993).

[3] C. Montag, N. D'Imperio, J. Kewisch, R. Lee, and T. Satogata, Proceedings of the EPAC 2002, Paris (to be published).

[4] M. Berz, É. Forest, and J. Irwin, Part. Accel. 24, 91-107 (1989).

[5] M. Berz, Part. Accel. 24, 109-124 (1989).

[6] F. Schmidt, CERN Report No. SL/94-56 (AP), 1994, http://home.cern.ch/frs/Documentation/doc.html

[7] H. Grote, SL Note No. 97-02 (AP), 1997.

[8] H. Grote and F. Schmidt, "The MAD-X Program" (to be published). This upgrade of the MAD- 8 code does include a module that converts MAD-X machine description into the SIXTRACK input files.

[9] É. Forest, The DALIE Code, 1986 (unpublished).

[10] H. Mais and C. Mari, in Proceedings of the CERN Accelerator School, Fifth General Accelerator Physics Course (CERN Report No. 94-01, 1994). 